\title{
Traveling-wave photomixer with recessed interdigitated contacts on low-temperature-grown GaAs
}

\author{
M. Mikulics ${ }^{\text {a) }}$ \\ Max-Planck-Institute for Radioastronomy, Bonn, D-53121 Bonn, Germany, and Institute of Thin Films and \\ Interfaces, Research Centre Jülich, D-52425 Jülich, Germany, and Institut für Hochfrequenztechnik, \\ Technische Universität Braunschweig, Schleinitzstraße 22, D-38106 Braunschweig, Germany \\ E. A. Michael, R. Schieder, and J. Stutzki \\ Physics Institute, University of Cologne, Zülpicher Str. 77, 50937 Köln, Germany \\ R. Güsten \\ Max-Planck-Institute for Radioastronomy, Bonn, D-53121 Bonn, Germany \\ M. Marso, A. van der Hart, H. P. Bochem, and H. Lüth \\ Institute of Thin Films and Interfaces and CNI - Center of Nanoelectronic Systems for Information \\ Technology, Research Centre Jülich, D-52425 Jülich, Germany \\ P. Kordoš \\ Institute of Electrical Engineering, Slovak Academy of Sciences, SK-84104 Bratislava, and Institute of \\ Microelectronics, Slovak Technical University, SK-81219 Bratislava, Slovak Republic
}

(Received 1 August 2005; accepted 5 December 2005; published online 26 January 2006)

\begin{abstract}
We have fabricated and characterized novel traveling-wave photomixers with recessed interdigitated metal-semiconductor-metal (MSM) contacts based on low-temperature-grown GaAs. The new recessed MSM geometry led to an improved electric-field distribution inside the photomixer structure and resulted in an up-to-100\% increase in the output power of continuously operated devices, compared to conventional MSM devices with standard surface electrodes fabricated on an identical material. The recessed electrode structure also resulted in lower saturation of output power at higher input powers, enabling it to take advantage of higher input powers. (C) 2006 American Institute of Physics. [DOI: 10.1063/1.2168250]
\end{abstract}

Molecular-beam epitaxial (MBE) GaAs grown at low temperature (LT-GaAs) has been recognized for more than a decade for its subpicosecond photocarrier trapping time and acceptable carrier mobility. It is therefore a common material for the fabrication of ultrafast photonic devices. ${ }^{1}$ The continuous-wave photomixer as a tunable source of terahertz $(\mathrm{THz})$ radiation is one particular example. ${ }^{2}$ However, the efficiency limits of the LT-GaAs material have already been reached, and therefore further improvement of the performance of LT-GaAs based photonic devices can only be obtained by optimization of the device structure. One such approach is using a AlAs/GaAs Bragg reflector below the LTGaAs active layer. ${ }^{3}$ If LT-GaAs photomixers are designed as small-area devices, their output power is mostly resistancecapacitance $(R C)$ limited. Recently, we reported a large-area traveling-wave photomixer design using a coplanar stripline with interdigitated finger contacts. ${ }^{4}$ It combines the advantages of a traveling-wave setup, which enables one to circumvent the device $R C$ constant $^{5}$ and of conventional interdigitated small-area devices resulting in an increase in the $\mathrm{THz}$ output power. ${ }^{4}$ The finger contacts were fabricated on the surface of the semiconducting material, as typically reported in previous published data on the metalsemiconductor-metal (MSM) photodetectors and photomixers. ${ }^{6-11}$ However, it is well known that conventional planar MSM structures possess a strongly nonuniform electric-field distribution between the contacts, with their

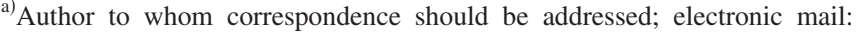
m.mikulics@fz-juelich.de
}

weakest part being in the central absorption region. Metal contacts on the sidewalls of etched trenches were used in the past for bandwidth enhancement on $\mathrm{Si}$ MSM photodetectors. $^{12}$

In this letter, we report on the fabrication and performance characterization of large-area traveling-wave photomixers with partially recessed interdigitated MSM finger contacts on LT-GaAs. We demonstrate that this new geometry results in a remarkable improvement in photomixer performance when compared to standard devices with nonrecessed surface contacts fabricated on the same LT-GaAs material. Higher output power and the suitability to operate at higher input powers make this approach suitable for highperformance ultrafast photonic devices, particularly photomixers.

Standard LT-GaAs MSM structures with surface contacts typically suffer from a nonuniform electric-field distribution, i.e., weaker light absorption, in the active layer and are prone to an electrical breakdown on the semiconductor surface. The basic idea behind our concept is to bury the finger contacts partially in the active material and thus modify the electrical-field distribution between the contacts. ${ }^{13}$ Conventional MBE LT-GaAs grown at $275^{\circ} \mathrm{C}$ and annealed in situ at $600{ }^{\circ} \mathrm{C}$ was used for the fabrication of photomixers. The "V" profile trenches for recessed finger contacts were formed using an anisotropic wet etch solution of $\mathrm{H}_{3} \mathrm{PO}_{4}: \mathrm{H}_{2} \mathrm{O}_{2}: \mathrm{H}_{2} \mathrm{O}(3: 1: 50){ }^{14-16}$ Coming from the surface contacts, it is apparent that recessed electrodes can collect photogenerated carriers more effectively, and therefore an increase in photocurrent can be observed. With too large a 


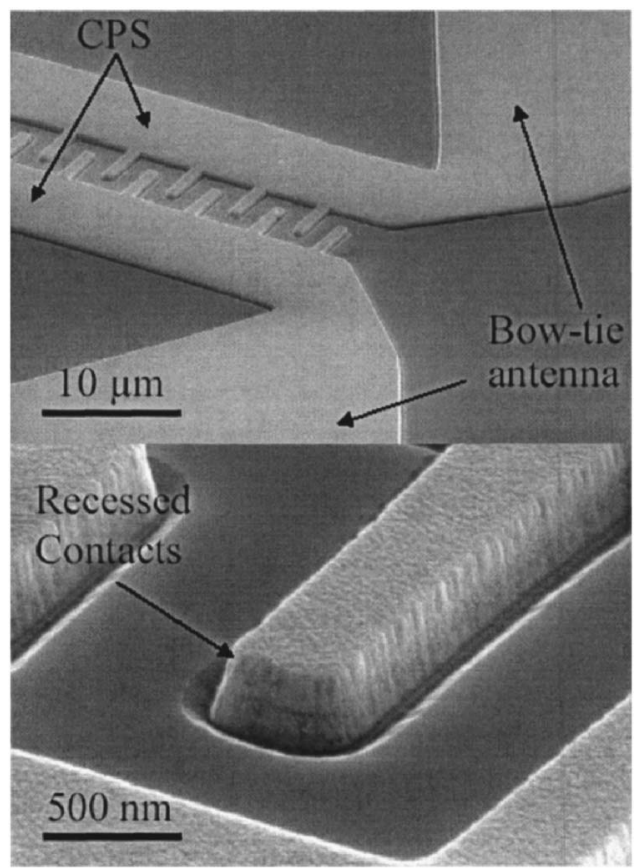

FIG. 1. Scanning electron microscope micrograph of recessed interdigitated finger contacts (bottom) integrated with a coplanar stripline in a travelingwave photomixer on LT-GaAs (top). The dimensions can be seen from the cross-sectional drawing. The contact width and spacing are $0.5 \mu \mathrm{m}$ and $1.4 \mu \mathrm{m}$, respectively, the recessed depth is $180 \mathrm{~nm}$.

contact depth, however, the total photocarrier collection efficiency decreases again due to the decreased electric-field strength at the semiconductor surface. On the basis of dc and pulsed response measurements on MSM photodetectors with etched trenches of different depths up to $300 \mathrm{~nm}$, we found that the optimal electrode depth - with respect to the collected photocurrent-is approximately $180 \mathrm{~nm} .{ }^{13}$ The photomixers consisted of a coplanar stripline integrated with recessed interdigitated finger contacts, as well as bowtie antenna $(70 \Omega)$ and contact pads. The $\mathrm{Ti} / \mathrm{Au}$ metallization on LT-GaAs was patterned using conventional electron-beam lithography and a lift-off technique. The finger width and finger spacing was $0.5 \mu \mathrm{m}$ and $1.4 \mu \mathrm{m}$, respectively. The coplanar stripline was $\sim 4 \mu \mathrm{m}$ wide. The finger contacts were integrated with the stripline on the length of $\sim 200 \mu \mathrm{m}$, as shown in Fig. 1. Thus, the described traveling-wave photomixers exhibited a large mixing area, with more than 100 finger contacts, compared to conventional small-area devices. For the sake of comparison, photomixers with the same geometry, but with nonrecessed surface electrodes, were fabricated on the same LT-GaAs material.

The $\mathrm{THz}$ power spectrum was measured with a calibrated magnetically tuned $4.2 \mathrm{~K} \mathrm{InSb}$ bolometer, which had a dip in the response around $0.5 \mathrm{THz}$ and a cutoff around 1.4 THz. Both features were not calibrated out from the frequency plot. Two heterodyned laser beams of different frequencies $(\sim 780 \mathrm{~nm}$ wavelength) were used for photomixer pumping. ${ }^{4}$ Figure 2 compares the output power versus frequency $\left(P_{\text {out }}-f\right)$ characteristics of a photomixer with recessed interdigitated contact against one with surface contacts. The total input power was $400 \mathrm{~mW}$ and the bias voltage was $15 \mathrm{~V}$. The recessed-contact photomixers exhibit about twice more output power in the frequency range up to $1 \mathrm{THz}$, in comparison to the mixers with surface contacts. The output power at $1 \mathrm{THz}$ of the recessed photomixer ex- tacts. Full lines are calculated.
Downloaded 21 Dec 2006 to 134.94.122.39. Redistribution subject to AlP license or copyright,

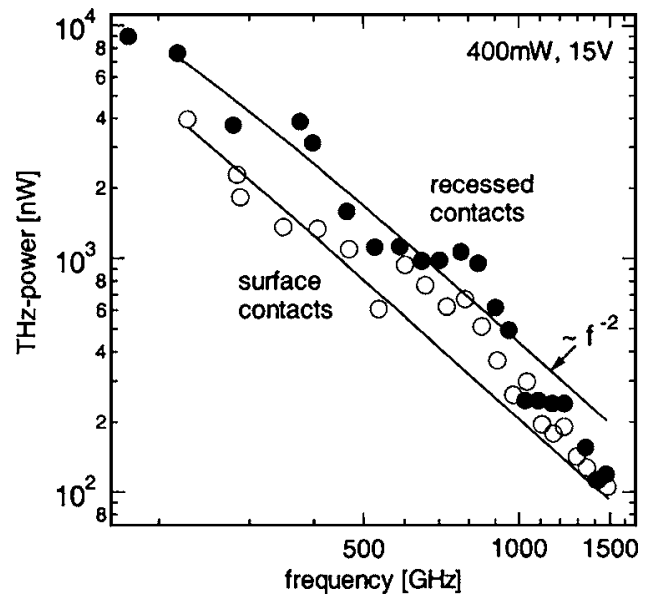

FIG. 2. THz output power spectrum for a traveling-wave photomixer with surface and recessed interdigitated MSM contacts of spacing $s=1.4 \mu \mathrm{m}$ fabricated on LT-GaAs (focus length $150 \mu \mathrm{m}$ full width at half maximum at input wavelength $780 \mathrm{~nm}$, input power $400 \mathrm{~mW}$, and bias voltage $15 \mathrm{~V}$ ).

hibits about a four times higher value than that previously reported for surface-based traveling-wave devices. ${ }^{5}$ The stronger rolloff of the recessed device beyond $1 \mathrm{THz}$ could be an artifact or due to the following: As the impedance match of the antenna to the stripline was optimized for the surface contacts, it might be nonoptimal for the recessed contacts at frequencies above $1 \mathrm{THz}$.

Figure 3 shows the output power as a function of the bias voltage for the photomixing frequency adjusted at 710 GHz. Typical data for the photomixers with recessed and surface contacts are presented. Better performance of the photomixer with recessed contacts in the whole range of applied bias voltages is evident. For lower biases (up to $\sim 7 \mathrm{~V}$ ), more than a $200 \%$ increase in the output power, when compared to the photomixer with surface contacts, is obtained. At higher biases, the output power of recessed photomixer increases moderately. Nevertheless, at the highest bias level tested $(15 \mathrm{~V})$, the output power of the recessed photomixer is still more than twice higher than that of the surface contacts photomixer. According to theory, the output power should increase with the bias as $P_{\mathrm{THz}}=K \times V_{b}^{2}$, where $K$ is the constant that depends on the incident optical power, emitted fre-

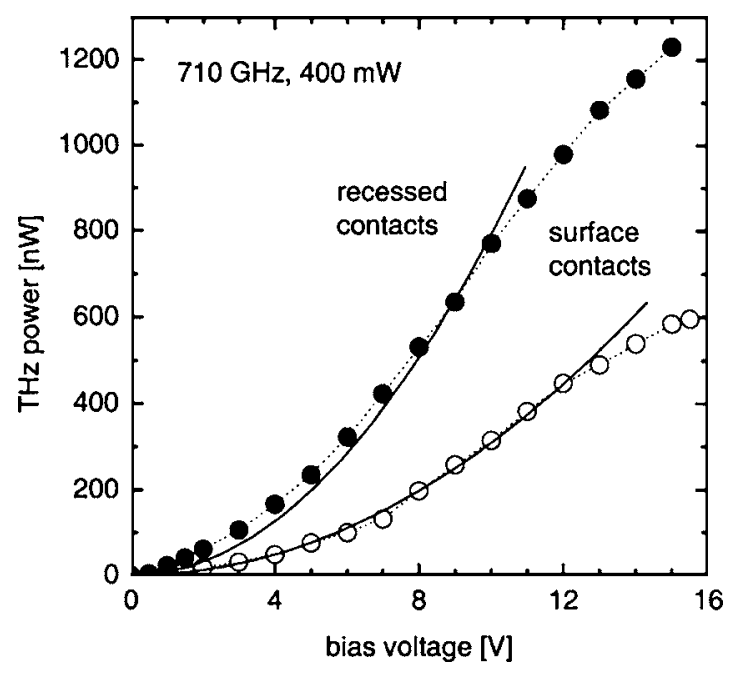

FIG. 3. Output power at $710 \mathrm{GHz}$ vs bias voltage for a traveling-wave photomixer on LT GaAs with surface and recessed interdigitated MSM contacts. Full lines are calculated. 


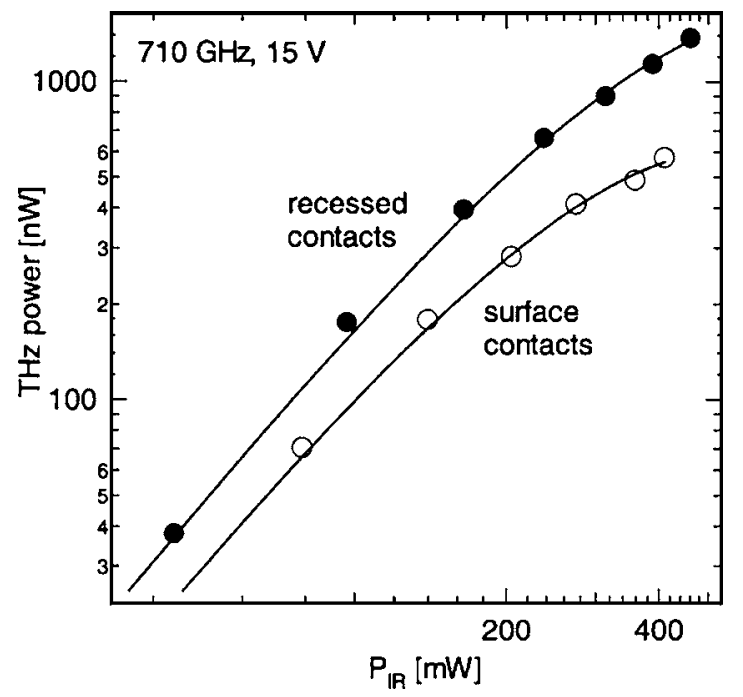

FIG. 4. Output power at $710 \mathrm{GHz}$ for a traveling-wave photomixer with surface and recessed interdigitated MSM contacts fabricated on LT-GaAs, measured as a function of input power (bias voltage $15 \mathrm{~V}$ ).

quency, radiation efficiency, carrier dynamics in LT-GaAs, number of finger contacts, and the length of the active region. ${ }^{2}$ Measured data for the recessed photomixer can be fitted according to this equation using $K=7.9 \times 10^{-9} \mathrm{~S}$ (full line in Fig. 3). For data on photomixers with surface contacts, we assume the same constant $K$ so that the measured data follow a $K\left(0.63 \times V_{b}\right)^{2}$ dependence, also shown in Fig. 3. This indicates a much weaker electric-field strength in the central absorption region than that in the recessed device at the same applied bias. Unfortunately, a more exact evaluation is difficult due to a strongly nonuniform electric-field distribution between the contacts. This is also partially true for recessed devices due to the space-charge region formed in the vicinity of the LT-GaAs/metal junction. ${ }^{17}$ Additional studies related to this observation are in progress.

Figure 4 depicts the output power as function of the infrared input power. In the whole measurement range, the photomixer with recessed contacts shows an almost factor of 2 increase of the output power, compared to the conventional surface device.

Since in the investigated traveling-wave photomixer structures a three times higher input power is distributed to about a one order of magnitude larger active area $\left(\sim 300 \mu \mathrm{m}^{2}\right)$ than in small-area structures, ${ }^{4}$ we did not observe the very strong saturation of output power at higher levels of the input power known from small-area design, which has been explained by overheating effects. ${ }^{18}$ The observed moderate saturation can be described by $P_{\text {in }}^{2} * \exp \left(-\right.$ const $\left.* P_{\text {in }}\right)$, which is clearly weaker and occurs later for the recessed contacts than for the surface contacts (Fig. 4).

In conclusion, we have fabricated LT-GaAs travelingwave photomixers with recessed interdigitated finger contacts and observed significant improvements in their output power, in comparison to standard surface electrode MSM structures. The recessed-contact MSM devices show up to $200 \%$-higher output power at low biases and up to $\sim 100 \%$ enhancement at higher bias levels. We can clearly associate this observed increase in the output power with increased electric-field strength in the central absorption region of recessed devices than that of conventional mixers with surface contacts. The traveling-wave photomixers with integrated recessed interdigitated finger contacts are suitable for use at higher input powers and bias voltages. It can therefore be concluded that they represent a novel type of LT-GaAs photomixer with enhanced performance.

${ }^{1}$ D. C. Look, Thin Solid Films 231, 61 (1993).

${ }^{2}$ E. R. Brown, F. W. Smith, and K. A. McIntosh, J. Appl. Phys. 73, 1480 (1993).

${ }^{3}$ J. E. Bjarnason, T. L. J. Chan, A. W. M. Lee, E. R. Brown, D. C. Driscoll, M. Hanson, A. C. Gossard, and R. E. Muller, Appl. Phys. Lett. 85, 3985 (2004).

${ }^{4}$ E. A. Michael, B. Vowinkel, R. Schieder, M. Mikulics, M. Marso, and P. Kordoš, Appl. Phys. Lett. 86, 111119 (2005).

${ }^{5}$ S. Matsuura, G. A. Blake, R. A. Wyss, J. C. Pearson, C. Kadow, A. W. Jackson, and A. C. Gossard, Appl. Phys. Lett. 74, 2872 (1999).

${ }^{6}$ P. Kordoš, A. Förster, M. Marso, and F. Ruders, Electron. Lett. 34, 119 (1998).

${ }^{7}$ E. Peytavit, S. Arscott, D. Lippens, G. Mouret, S. Matton, P. Masselin, R. Bocquet, J. F. Lampin, L. Desplanque, and F. Mollot, Appl. Phys. Lett. 81, 1174 (2002).

${ }^{8}$ S. Verghese, K. A. McIntosh, and E. R. Brown, IEEE Trans. Microwave Theory Tech. 45, 1301 (1997).

${ }^{9}$ T. Kleine-Ostmann, P. Knobloch, M. Koch, S. Hoffman, M. Breede, M. Hofmann, G. Hein, K. Pierz, M. Sperling, and K. Donhuijsen, Electron. Lett. 37, 1461 (2001).

${ }^{10}$ S. M. Duffy, S. Verghese, K. A. McIntosh, A. Jackson, A. C. Gossard, and S. Matsuura, IEEE Trans. Microwave Theory Tech. 49, 1032 (2001).

${ }^{11}$ I. S. Gregory, C. Baker, W. R. Tribe, I. V. Bradley, M. J. Evans, E. H. Linfield, A. G. Davies, and M. Missous, IEEE J. Quantum Electron. 41, 717 (2005).

${ }^{12}$ J. Y. L. Ho, and K. S. Wong, IEEE Photonics Technol. Lett. 8, 1064 (1996).

${ }^{13}$ M. Mikulics, Ph.D. thesis, RWTH Aachen and FZ Jülich, 2005.

${ }^{14}$ D. Gregušová, P. Eliaš, L. Malacky, R. Kúdela, and J. Škriniarová, Phys. Status Solidi A 151, 113 (1995).

${ }^{15}$ J. Škriniarová, J. Ková, J. Breza, and D. Gregušová, J. Electr. Eng. 48, 85 (1997).

${ }^{16}$ D. Gregušová, V. Cambel, J. Fedor, R. Kúdela, J. Soltýs, T. Lalinský, I. Kostic, and S. J. Bending, Appl. Phys. Lett. 82, 3704 (2003).

${ }^{17}$ P. Kordoš, M. Marso, A. Förster, J. Darmo, J. Betko, and G. Nimtz, Appl. Phys. Lett. 71, 1118 (1997)

${ }^{18}$ A. W. Jackson, Ph.D. thesis, University of California at Santa Barbara, 1999. 